\title{
Suggested new breakpoints of anti-MERS-CoV antibody ELISA titers: performance analysis of serologic tests
}

\author{
J.-H. Ko ${ }^{1,2}$ • M. A. Müller ${ }^{3,4}$ • H. Seok ${ }^{1}$ - G. E. Park ${ }^{1}$ - J. Y. Lee ${ }^{1}$ - S. Y. Cho ${ }^{1}$ • Y. E. Ha ${ }^{1}$ • \\ J. Y. Baek ${ }^{5}$ - S. H. Kim ${ }^{5}$ • J.-M. Kang ${ }^{6}$ - Y.-J. Kim ${ }^{6}$ • I. J. Jo ${ }^{7}$ - C. R. Chung ${ }^{8}$ • \\ M.-J. Hahn ${ }^{9}$ • C. Drosten ${ }^{3,4}$ • C.-I. Kang ${ }^{1}$ - D. R. Chung ${ }^{1,5}$ • J.-H. Song ${ }^{1,5}$ • E.-S. Kang ${ }^{10}$ • \\ K. R. Peck ${ }^{1}$
}

Received: 28 April 2017 / Accepted: 4 June 2017 /Published online: 11 July 2017

(C) Springer-Verlag GmbH Germany 2017

\begin{abstract}
To provide optimal cut-off values of anti-Middle East respiratory syndrome coronavirus (MERS-CoV) serologic tests, we evaluated performance of ELISA IgG, ELISA IgA, IFA IgM, and IFA IgG using 138 serum samples of 49 MERS-CoV-infected patients and 219 serum samples of 219 rRT-PCR-negative MERS-CoV-exposed healthcare personnel and patients. The performance analysis was conducted for two different purposes: (1) prediction of neutralization activity in MERS-CoV-infected patients, and (2) epidemiologic surveillance of MERS-CoV infections among MERS-CoV-exposed individuals. To evaluate performance according to serum
\end{abstract}

Jae-Hoon Ko and Marcel A. Müller contributed equally to this article as first authors.

Eun-Suk Kang and Kyong Ran Peck contributed equally to this article

Electronic supplementary material The online version of this article (doi:10.1007/s10096-017-3043-3) contains supplementary material, which is available to authorized users.

\section{E.-S. Kang}

eskang@skku.edu

$\triangle$ K. R. Peck

krpeck@skku.edu

1 Division of Infectious Diseases, Department of Medicine, Samsung Medical Center, Sungkyunkwan University School of Medicine, 81 Irwon-ro, Gangnam-gu, Seoul 135-710, Republic of Korea

2 Present address: Division of Infectious Diseases, Department of Internal Medicine, Armed Forces Capital Hospital, Seongnam, South Korea

3 Institute of Virology, Charité - Universitätsmedizin Berlin, Helmut-Ruska-Haus Charitéplatz 1, 10117 Berlin, Germany

4 German Centre for Infection Research, Braunschweig, Germany

5 Asia Pacific Foundation for Infectious Diseases (APFID), Seoul, Republic of Korea collection time, we used 'days post onset of illness (dpoi)' and 'days post exposure (dpex)' assessing neutralization activity and infection diagnosis, respectively. Performance of serologic tests improved with delayed sampling time, being maximized after a seroconversion period. In predicting neutralization activity, ELISA IgG tests showed optimal performance using sera collected after 21 dpoi at cut-off values of OD ratio 0.4 (sensitivity $100 \%$ and specificity $100 \%$ ), and ELISA IgA showed optimal performance using sera collected after 14 dpoi at cut-off value of OD ratio 0.2 (sensitivity $85.2 \%$ and specificity $100 \%$ ). In diagnosis of MERS-CoV
6 Division of Infectious Diseases, Department of Pediatrics, Samsung Medical Center, Sungkyunkwan University School of Medicine, Seoul, Republic of Korea

7 Department of Emergency Medicine, Samsung Medical Center, Sungkyunkwan University School of Medicine, Seoul, Republic of Korea

8 Department of Critical Care Medicine, Samsung Medical Center, Sungkyunkwan University School of Medicine, Seoul, Republic of Korea

9 Department of Molecular Cell Biology, Center for Molecular Medicine, Samsung Biomedical Research Institute, Sungkyunkwan University School of Medicine, Suwon 440-746, South Korea

10 Department of Laboratory Medicine and Genetics, Samsung Medical Center, Sungkyunkwan University School of Medicine, Seoul, Republic of Korea 
infection, ELISA IgG exhibited optimal performance using sera collected after $28 \mathrm{dpex}$, at a cut-off value of OD ratio 0.2 (sensitivity $97.3 \%$ and specificity $92.9 \%$ ). These new breakpoints are markedly lower than previously suggested values (ELISA IgG OD ratio 1.1, sensitivity $34.8 \%$ and specificity $100 \%$ in the present data set), and the performance data help serologic tests to be practically used in the field of MERS management.

Keywords Middle East respiratory syndrome coronavirus . Serology $\cdot$ Antibody $\cdot$ Sensitivity $\cdot$ Specificity

\section{Introduction}

Middle East respiratory syndrome coronavirus (MERS-CoV) is a novel beta coronavirus that may cause lethal respiratory disease [1]. Anti-MERS-CoV serologic tests including enzyme-linked immunosorbent assay (ELISA) and immunofluorescence assay (IFA) are available as commercial kits, and have been used for various purposes including epidemiologic investigations, evaluation of antibody kinetics, and assessing feasibility of convalescent plasma infusion therapy [2-6]. The manufacturer's instructions provided relatively high cut-off values for positivity to warrant specificity, which were derived from limited positive samples (ELISA IgG, Euroimmun, Lübeck, Germany; 4 MERS patient samples and 500 negative controls) [7]. In previously conducted serologic studies, cutoff values were differently applied depending on the purpose of serologic tests: several epidemiologic studies applied low cut-off values to increase sensitivity of the test $[3,6]$, while others followed the manufacturer's instructions [2,4]. Also, as not all confirmed MERS-CoV-infected patients mounted robust neutralization activity [2], the performance of serologic tests should be separately analyzed depending on the purposes of the tests: predicting neutralization activity in MERS-CoVinfected patients and diagnosing MERS-CoV infection in epidemiologic surveillance. To provide optimal cut-off values depending on the purposes of the tests, we evaluated performance of various anti-MERS-CoV serologic tests using 138 serum samples of 49 MERS-CoV-infected patients and 219 serum samples of 219 rRT-PCR-negative MERS-CoV-exposed healthcare personnel (HCP) and patients.

\section{Methods}

\section{Performance analysis depending on the purposes of serologic tests}

The performance analysis included sensitivity, specificity, positive predictive value, and negative predictive value, and was conducted under two different purposes of serologic tests:
(1) prediction of neutralization activity in MERS-CoVinfected patients, and (2) epidemiologic surveillance of MERS-CoV infections among MERS-CoV-exposed individuals. Plaque reduction neutralization test (PRNT) and sputum real-time reverse transcriptase polymerase chain reaction (rRT-PCR) for MERS-CoV were used as gold standards for assessing neutralization activity and diagnosing MERS-CoV infection, respectively.

MERS-CoV exposure dates and symptom onsets of collected serum samples were clearly identified owing to thorough contact investigation and monitoring of exposed individuals [8]. To provide a common point of reference, we used 'days post onset of illness (dpoi)' assessing neutralization activity since symptom onset could be clearly identified among MERS-CoV-infected patients. Meanwhile, as epidemiologic surveillances are usually conducted on the basis of exposure events, we applied 'days post exposure (dpex)' assessing diagnostic performance of serologic tests in epidemiologic surveillance.

To compare performances of serologic tests depending on serum collection time, each test was evaluated at three different timepoints: (1) regardless of serum collection time, (2) after 14 dpoi (or 21 dpex), and (3) after $21 d p o i$ (or 28 dpex).

\section{Study population and samples}

During the 2015 Korean MERS outbreak, we obtained 138 serum samples from 49 MERS-CoV infected patients. Study population included 42 patients who were managed at a 1950 bed tertiary care university hospital $[8,9]$, and seven patients who donated sera for plasma infusion therapy or serologic testing. MERS-CoV infections were confirmed on the basis of rRTPCR assays targeting upstream of the E gene (upE) and the open-reading frame gene 1a (ORF1a) [10, 11]. Epidemiologic investigation data and electronic medical records were reviewed to obtain exact exposure date and symptom onset. One or two serum samples were collected per week of illness during hospitalization. Follow-up serum samples were obtained up to 6 months from symptom onset at an outpatient clinic. Sera of 219 rRT-PCR-negative MERS-CoV-exposed HCP and patients were used as negative control samples [3]. Collected samples were stored at $-70{ }^{\circ} \mathrm{C}$ for about three months before testing. The Institutional Review Board of Samsung Medical Center approved the present study.

\section{Serologic tests for anti-MERS-CoV antibody}

\section{ELISA $\operatorname{Ig} G$ and $\operatorname{IgA}$}

Anti-MERS-CoV ELISA IgG and IgA (Euroimmun, Lübeck, Germany) were based on soluble MERS-CoV spike protein S1 domain expressed in HEK-293 T cells $[6,12-14]$. Sera were tested according to the 
manufacturer's instructions with 1:100 dilutions. Secondary detection was done with peroxidase-labeled anti-human $\operatorname{IgG}$ and $\operatorname{IgA}$. ELISA IgG was initially tested in all of the collected serum samples, and other serologic tests including ELISA IgA, IFA, and PRNT were selectively performed depending on ELISA IgG (using optical density (OD) ratio cut-off value of 0.2 ) and sputum rRTPCR results (Table 1). Since IFA IgG was limitedly tested among an rRT-PCR-negative population, diagnostic performance for epidemiologic surveillance of IFA IgG could not be evaluated.

\section{IFA $\operatorname{Ig} G$ and $\operatorname{Ig} M$}

Anti-MERS-CoV IFA IgG and IgM (Euroimmun) were performed with slides carrying Vero cells infected with full MERS-CoV [12, 14, 15]. Sera were tested according to the manufacturer's instructions with 1:10 and 1:100 dilutions for IFA IgM and IgG, respectively.

For comparison of the performance of IFA IgG with that of ELISA IgG, 71 sera were selected for titration from 1:50 to
1:1000 dilutions, including sera collected between 14 and 27 $d p o i$ (presumed window period of seroconversion) $[4,5]$, and sera of patients who were serially sampled at least four times (Table 1).

\section{PRNT}

MERS-CoV PRNT was performed as previously described $[12,14]$. Pre-dilution before setting up the log2-dilution series was 1:10, defining 1:20 as the lowest possible significant titer for categorizing a sample as positive [12].

\section{Statistical analysis}

Cut-off values with optimal sensitivity and specificity were analyzed per 0.1 OD ratio or each IFA intensity. Areas under the curve (AUCs) were calculated using the receiver operating characteristic (ROC) curve. R-3.3.1 for Windows (RStudio, Boston, MA, USA) was used for all statistical analyses.
Table 1 Number of individuals and serum samples that underwent serologic testing

\begin{tabular}{|c|c|c|c|c|c|c|}
\hline \multirow{2}{*}{$\begin{array}{l}\text { Performance } \\
\text { analysis }\end{array}$} & \multirow[t]{2}{*}{ Number of sera and patients } & \multicolumn{5}{|c|}{ Anti-MERS-CoV serologic tests } \\
\hline & & $\begin{array}{l}\text { ELISA } \\
\text { IgG }\end{array}$ & $\begin{array}{l}\text { ELISA } \\
\text { IgA }\end{array}$ & $\begin{array}{l}\text { IFA } \\
\text { IgM }\end{array}$ & $\begin{array}{l}\text { IFA } \\
\text { IgG }\end{array}$ & PRNT \\
\hline $\begin{array}{l}\text { Prediction of } \\
\text { neutralization } \\
\text { activity }\end{array}$ & $\begin{array}{l}138 \text { sera from } 49 \text { MERS-CoV-infected } \\
\text { patients }\end{array}$ & $\mathrm{O}$ & $\mathrm{O}$ & $\mathrm{O}^{*}$ & $\mathrm{O}^{\dagger}$ & $\mathrm{O}$ \\
\hline \multirow[t]{4}{*}{$\begin{array}{l}\text { Epidemiologic } \\
\text { surveillance }\end{array}$} & $\begin{array}{l}158 \text { sera from } 158 \text { MERS-CoV-exposed, } \\
\text { rRT-PCR-negative HCP, ELISA IgG } \\
\text { OD ratio }<0.2\end{array}$ & $\mathrm{O}$ & $\mathrm{X}$ & $\mathrm{X}$ & $\mathrm{X}$ & $\mathrm{X}$ \\
\hline & $\begin{array}{l}11 \text { sera from } 11 \text { MERS-CoV-exposed, } \\
\text { rRT-PCR-negative HCP, ELISA IgG } \\
\text { OD ratio } \geq 0.2\end{array}$ & $\mathrm{O}$ & $\mathrm{O}$ & $\mathrm{O}$ & $\mathrm{O}^{\ddagger}$ & $\mathrm{O}$ \\
\hline & $\begin{array}{l}43 \text { sera from } 43 \text { MERS-CoV-exposed, } \\
\text { rRT-PCR-negative patients, ELISA } \\
\text { IgG OD ratio }<0.2\end{array}$ & $\mathrm{O}$ & $\mathrm{X}$ & $\mathrm{O}$ & $\mathrm{X}$ & $\mathrm{X}$ \\
\hline & $\begin{array}{l}7 \text { sera from } 7 \text { MERS-CoV-exposed, } \\
\text { rRT-PCR-negative patients, ELISA } \\
\text { IgG OD ratio } \geq 0.2\end{array}$ & $\mathrm{O}$ & $\mathrm{O}$ & $\mathrm{O}$ & $\mathrm{O}$ & $\mathrm{O}$ \\
\hline \multicolumn{2}{|c|}{ Total tested serum samples } & 357 & 156 & 194 & 89 & 156 \\
\hline \multicolumn{2}{|c|}{ Total tested individuals } & 268 & 67 & 110 & 48 & 67 \\
\hline
\end{tabular}

ELISA IgG was initially tested in all collected samples, and other serologic tests were selectively performed depending on rRT-PCR and ELISA IgG results. A 0.2 OD ratio cut-off value was applied for ELISA, which was approximately three-fold compared with the median (0.06) value of 41 healthy individuals

${ }^{*}$ Five sera that were collected late were not tested. ${ }^{\dagger}$ To compare performance in predicting neutralizing activity with ELISA IgG, anti-MERS-CoV IFA IgG was tested with titration from 1:50 to 1:1000 dilutions in selected sera of MERS-CoV-infected patients: 18 sera from 18 patients collected between 14 and 27 dpoi (presumed window period of seroconversion) and 53 sera from 12 patients whose sera were serially collected at least four times. ${ }^{\ddagger}$ To substantiate ELISA results, IFA IgG was tested in these samples at a 1:100 dilution, which was not included in performance analysis.

MERS-CoV Middle East respiratory syndrome coronavirus, ELISA enzyme-linked immunosorbent assay, IFA immunofluorescence assay, $P R N T$ plaque reduction neutralization test, $r R T-P C R$ real-time reverse transcriptase polymerase chain reaction, $H C P$ healthcare personnel, $O D$ optical density, dpoi days post onset of illness 


\section{Results}

\section{Performance of serologic tests in predicting neutralization activity}

The performance of serologic tests improved with delayed sampling time, being maximized when sera collected after 21 dpoi were used (Fig. 1a). In predicting neutralization activity, ELISA IgG and IFA IgM tests showed optimal performance using sera collected after $21 \mathrm{dpoi}$, at cut-off values of OD ratio 0.4 and weekly-positive IFA intensity, respectively (Table 2). Especially, ELISA IgG showed $100 \%$ sensitivity and $100 \%$ specificity at this time point, while ELISA IgG and IFA IgM exhibited slightly lower performance (area under the curve (AUC) 1.000, 0.996, and 0.917, respectively). Meanwhile, ELISA IgA showed optimal performance using sera collected after $14 d p o i$ at cut-off value of OD ratio 0.2. Detailed performance values depending on serum collection time and cut-off values are presented in Supplementary Tables 1 to 3 . IFA IgG showed optimal performance with cut-off value of 1:500 dilutions, and overall performance was not superior to ELISA IgG (Supplementary Table 4).

a

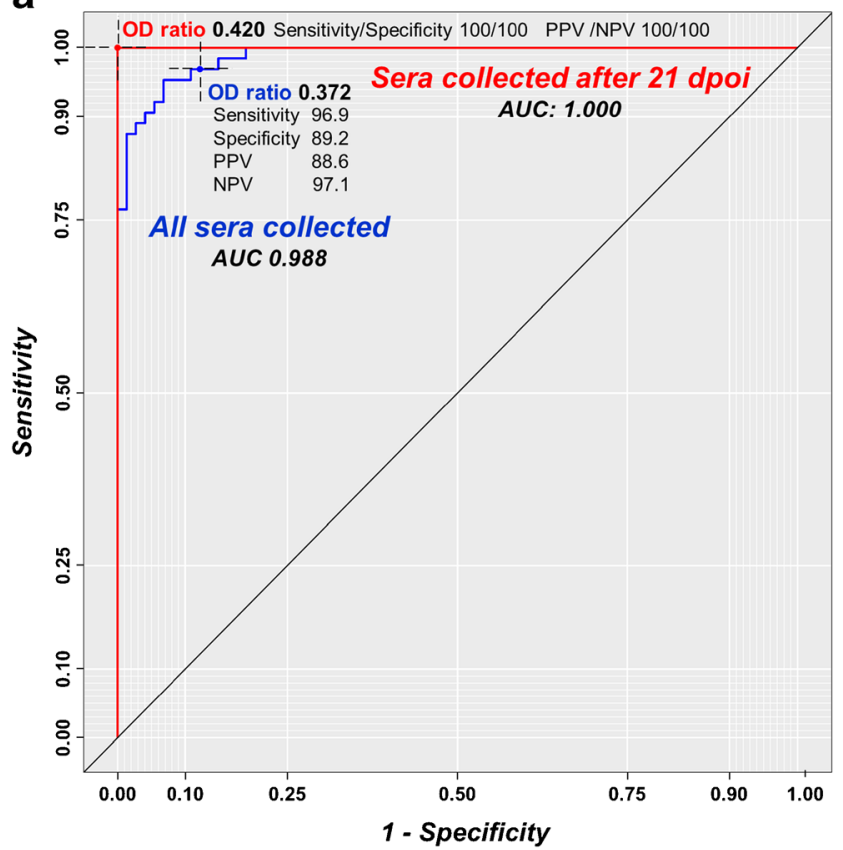

Fig. 1 Changes in ROC curves of anti-MERS-CoV ELISA IgG antibodies for prediction of neutralizing activity and diagnosis of MERS-CoV infection depending on serum collection time. (a) ROC curve of ELISA IgG OD ratios predicting neutralization activity in MERS-CoV-infected patients. When sera collected after 21 dpoi were used, both sensitivity and specificity increased to $100 \%$. An ELISA OD ratio of 0.420 was the best cut-off value based on the ROC curve, and 0.4 was the optimal value on the basis of a 0.1 OD ratio. (b) ROC curve of ELISA IgG OD ratios for the diagnosis of MERS-CoV infection in a
Performance of serologic tests in diagnosing MERS-CoV infection

In diagnosing MERS-CoV infection, ELISA IgG, ELISA IgA, and IFA IgM tests showed optimal performance using sera collected after 28 dpex, at cut-off value of OD ratio 0.2 , OD ratio 0.2 , and weakly-positive IFA intensity, respectively (Table 3). Most values of rRT-PCRnegative MERS-CoV-exposed individuals were under these cut-off values, discriminating MERS-CoV-infected and non-infected individuals (Fig. 2). Overall, ELISA IgG showed better performance than ELISA IgA or IFA IgM for diagnosing MERS-CoV infection (AUC 0.982, 0.914 , and 0.875 , respectively, when sera collected after 28 dpex were used). Performance of serologic tests improved with delayed sampling time, being maximized when sera collected after 28 dpex were used (Fig. 1b and Supplementary Tables 5 to 7). In addition, the specificity of ELISA IgG, ELISA IgA, and IFA IgM tests was $100 \%$ regardless of serum collection time, at cut-off value of $\mathrm{OD}$ ratio 0.5 , OD ratio 0.3 , and IFA intensity $1+$, respectively.

\section{b}

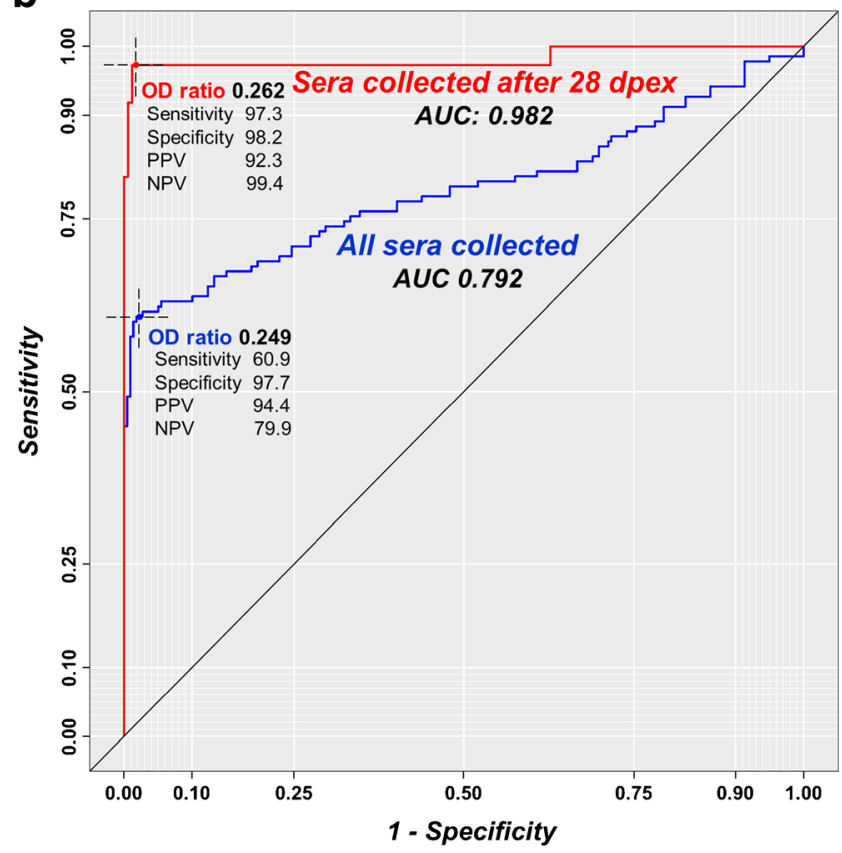

MERS-CoV-exposed population. When sera collected after 28 dpex were used, sensitivity and specificity increased to 97.3 and 98.2, respectively. An ELISA OD ratio of 0.262 was the best cut-off value based on the ROC curve, and 0.2 was the optimal value on the basis of a 0.1 OD ratio. $R O C$ receiver operating characteristic, $M E R S-C o V$ Middle East respiratory syndrome coronavirus, ELISA enzyme-linked immunosorbent assay, $O D$ optical density, dpoi days post onset of illness, dpex days post exposure 
Table 2 Performance of anti-MERS-CoV antibody tests in predicting neutralization activity

\begin{tabular}{|c|c|c|c|c|c|}
\hline \multirow[b]{2}{*}{ Purpose of test } & \multicolumn{2}{|c|}{ Test methods } & \multirow{2}{*}{\multicolumn{3}{|c|}{ Cut-off values for positivity }} \\
\hline & Sampling time & $\begin{array}{l}\text { Predictive } \\
\text { values }\end{array}$ & & & \\
\hline \multirow{27}{*}{$\begin{array}{l}\text { Prediction of } \\
\text { neutralization } \\
\text { activity in MERS- } \\
\text { CoV-infected } \\
\text { patients }\end{array}$} & ELISA IgG & & $\geq 0.3$ & $\geq 0.4$ & $\geq 0.6$ \\
\hline & All collected & Sensitivity & $100 \%$ & $95.3 \%$ & $87.5 \%$ \\
\hline & sera regardless & Specificity & $79.7 \%$ & $91.9 \%$ & $97.3 \%$ \\
\hline & of sampling & PPV & $81.0 \%$ & $91.0 \%$ & $96.6 \%$ \\
\hline & $A U C=0.988$ & NPV & $100 \%$ & $95.8 \%$ & $90.0 \%$ \\
\hline & Sera collected & Sensitivity & $100 \%$ & $100 \%$ & $93.9 \%$ \\
\hline & after 21 dpoi & Specificity & $87.5 \%$ & $100 \%$ & $100 \%$ \\
\hline & $\begin{array}{l}(\mathrm{n}=41) \\
(J C=100 n\end{array}$ & PPV & $97.1 \%$ & $100 \%$ & $100 \%$ \\
\hline & & NPV & $100 \%$ & $100 \%$ & $80.0 \%$ \\
\hline & ELISA IgA & & $\geq 0.1$ & $\geq 0.2$ & $\geq 0.3$ \\
\hline & All the collected & Sensitivity & $98.4 \%$ & $84.4 \%$ & $75.0 \%$ \\
\hline & sera regardless & Specificity & $74.3 \%$ & $98.6 \%$ & $100 \%$ \\
\hline & $\begin{array}{l}\text { of sampling } \\
\text { time }(n=138)\end{array}$ & PPV & $76.8 \%$ & $98.2 \%$ & $100 \%$ \\
\hline & $\begin{array}{l}\text { time }(\mathrm{n}=138) \\
\boldsymbol{A} \boldsymbol{C}=\mathbf{0 . 9 8 2}\end{array}$ & NPV & $98.2 \%$ & $88.0 \%$ & $82.2 \%$ \\
\hline & Sera collected & Sensitivity & $100 \%$ & $85.2 \%$ & $75.4 \%$ \\
\hline & after $14 d p o i$ & Specificity & $75.0 \%$ & $100 \%$ & $100 \%$ \\
\hline & $(\mathrm{n}=77)$ & PPV & $93.8 \%$ & $100 \%$ & $100 \%$ \\
\hline & $A U C=0.998$ & NPV & $100 \%$ & $64.0 \%$ & $54.6 \%$ \\
\hline & IFA IgM & & $\geq \mathbf{w}+$ & $\geq 1+$ & $\geq 2+$ \\
\hline & All collected & Sensitivity & $85.2 \%$ & $75.4 \%$ & $57.4 \%$ \\
\hline & sera regardless & Specificity & $77.8 \%$ & $91.7 \%$ & $97.2 \%$ \\
\hline & of sampling & PPV & $76.5 \%$ & $88.5 \%$ & $94.6 \%$ \\
\hline & $\begin{array}{l}\text { time }(\mathrm{n}=133) \\
\boldsymbol{A U} \boldsymbol{C}=\mathbf{0 . 8 7 8}\end{array}$ & NPV & $86.2 \%$ & $81.5 \%$ & $72.9 \%$ \\
\hline & Sera collected & Sensitivity & $83.3 \%$ & $73.3 \%$ & $56.7 \%$ \\
\hline & after $21 d p o i$ & Specificity & $100 \%$ & $100 \%$ & $100 \%$ \\
\hline & & PPV & $100 \%$ & $100 \%$ & $100 \%$ \\
\hline & $A U C=0.917$ & NPV & $54.5 \%$ & $42.9 \%$ & $31.6 \%$ \\
\hline
\end{tabular}

Data are expressed as a percentage of each predictive value according to various cut-off values. Cut-off values with optimal sensitivity and specificity analyzed per 0.1 OD ratio (ELISA) or IFA intensity are presented as gray-scale. AUCs were calculated from the ROC curve. The population of this analysis is 49 MERS-CoV-infected patients confirmed by rRT-PCR (Table 1). The neutralization activity of sera was confirmed by PRNT and a 1:20 dilution was defined as the lowest significant titer.

MERS-CoV Middle East respiratory syndrome coronavirus, ELISA enzyme-linked immunosorbent assay, AUC area under the curve, $P P V$ positive predictive value, $N P V$ negative predictive value, $d p o i$ days post onset of illness, IFA immunofluorescence assay, $w+$ weak positive, $O D$ optical density, $R O C$ receiver operating characteristic, $r R T-P C R$ real-time reverse transcriptase polymerase chain reaction, $P R N T$ plaque reduction neutralization test

\section{Discussion}

Neutralization testing is a gold standard method for detecting antiviral antibodies, especially exhibiting virus-killing function. However, neutralization tests against MERS-CoV cannot be readily performed worldwide as it requires biosafety level 3 facilities, skilled experts, and carries a potential risk of infection [5]. To reduce such risks and workloads, previous anti-MERS-CoV serologic studies applied step-wise approaches using ELISA and IFA, which are relatively easy and safe to test $[6,12]$. To make anti-MERS-CoV serologic tests more practical, we assessed performance of ELISA and IFA tests for two clinical purposes and suggested optimal cutoff values. 
Table 3 Performance of anti-MERS-CoV antibody tests in epidemiologic surveillance of MERS-CoV infection

\begin{tabular}{|c|c|c|c|c|c|}
\hline \multirow[b]{2}{*}{ Purpose of test } & \multicolumn{2}{|c|}{ Test methods } & \multirow{2}{*}{\multicolumn{3}{|c|}{ Cut-off values for positivity }} \\
\hline & Sampling time & $\begin{array}{l}\text { Predictive } \\
\text { values }\end{array}$ & & & \\
\hline \multirow{27}{*}{$\begin{array}{l}\text { Epidemiologic } \\
\text { surveillance of } \\
\text { MERS-CoV } \\
\text { infections among } \\
\text { MERS-CoV-exposed } \\
\text { individuals }\end{array}$} & ELISA IgG & & $\geq 0.2$ & $\geq 0.3$ & $\geq 0.5$ \\
\hline & All collected & Sensitivity & $63.0 \%$ & $57.2 \%$ & $44.9 \%$ \\
\hline & sera regardless & Specificity & $94.1 \%$ & $99.1 \%$ & $100 \%$ \\
\hline & of sampling & PPV & $87.0 \%$ & $97.5 \%$ & $100 \%$ \\
\hline & $\begin{array}{l}\boldsymbol{A} \boldsymbol{U} \boldsymbol{C}=\mathbf{0 . 7 9 2}\end{array}$ & NPV & $80.2 \%$ & $78.6 \%$ & $74.2 \%$ \\
\hline & Sera collected & Sensitivity & $97.3 \%$ & $89.2 \%$ & $81.1 \%$ \\
\hline & after 28 dpex & Specificity & $92.9 \%$ & $99.4 \%$ & $100 \%$ \\
\hline & $(\mathrm{n}=206)$ & PPV & $75.0 \%$ & $97.1 \%$ & $100 \%$ \\
\hline & $A U C=0.982$ & NPV & $99.4 \%$ & $97.7 \%$ & $96.0 \%$ \\
\hline & ELISA IgA & & $\geq 0.1$ & $\geq 0.2$ & $\geq 0.3$ \\
\hline & All collected & Sensitivity & $59.4 \%$ & $39.9 \%$ & $34.8 \%$ \\
\hline & sera regardless & Specificity & $77.8 \%$ & $88.9 \%$ & $100 \%$ \\
\hline & of sampling & PPV & $95.3 \%$ & $96.5 \%$ & $100 \%$ \\
\hline & $\begin{array}{l}\text { time }(\mathrm{n}=156) \\
\boldsymbol{A} \boldsymbol{U} \boldsymbol{C}=\mathbf{0 . 7 8 9}\end{array}$ & NPV & $20.0 \%$ & $16.2 \%$ & $16.7 \%$ \\
\hline & Sera collected & Sensitivity & $86.5 \%$ & $73.0 \%$ & $62.2 \%$ \\
\hline & after $28 d p e x$ & Specificity & $75.0 \%$ & $91.7 \%$ & $100 \%$ \\
\hline & $(\mathrm{n}=49)$ & PPV & $91.4 \%$ & $96.4 \%$ & $100 \%$ \\
\hline & $A U C=0.914$ & NPV & $64.3 \%$ & $52.4 \%$ & $46.2 \%$ \\
\hline & IFA IgM & & $\geq \mathbf{w}+$ & $\geq 1+$ & $\geq 2+$ \\
\hline & All collected & Sensitivity & $51.9 \%$ & $39.1 \%$ & $27.8 \%$ \\
\hline & sera regardless & Specificity & $96.7 \%$ & $100 \%$ & $100 \%$ \\
\hline & $\begin{array}{l}\text { of sampling } \\
\text { time }(n=194)\end{array}$ & PPV & $97.2 \%$ & $100 \%$ & $100 \%$ \\
\hline & $\begin{array}{l}\boldsymbol{A} \boldsymbol{U} \boldsymbol{C}=\mathbf{0 . 7 4 9}\end{array}$ & NPV & $48.0 \%$ & $43.0 \%$ & $38.9 \%$ \\
\hline & Sera collected & Sensitivity & $75.0 \%$ & $65.6 \%$ & $50.0 \%$ \\
\hline & after 28 dpex & Specificity & $100 \%$ & $100 \%$ & $100 \%$ \\
\hline & & PPV & $100 \%$ & $100 \%$ & $100 \%$ \\
\hline & $A U C=0.875$ & NPV & $60.0 \%$ & $52.2 \%$ & $42.9 \%$ \\
\hline
\end{tabular}

Data are expressed as a percentage of each predictive value according to various cut-off values. Cut-off values with optimal sensitivity and specificity analyzed per 0.1 OD ratio (ELISA) or IFA intensity are presented as gray-scale. AUCs were calculated from the ROC curve. The population this analysis is 268 MERS-CoV-exposed individuals (Table 1). Diagnosis of MERS-CoV infection was confirmed by positive rRT-PCR assay of respiratory specimens.

MERS-CoV Middle East respiratory syndrome coronavirus, ELISA enzyme-linked immunosorbent assay, AUC area under the curve, PPV positive predictive value, $N P V$ negative predictive value, dpex days post exposure, IFA immunofluorescence assay, $w+$ weak positive, $O D$ optical density, $R O C$ receiver operating characteristic, $r R T-P C R$ real-time reverse transcriptase polymerase chain reaction

As previous cut-off values for positivity were provided for diagnosis of MERS-CoV infection [7], we firstly suggest cut-off values for predicting neutralization activity. In the present analysis, both ELISA IgG and IgA excellently predicted neutralization activity (using sera collected after 21 dpoi, AUC 1.000 and 0.996, respectively). Of note, ELISA IgG showed $100 \%$ sensitivity and $100 \%$ specificity at this time point, with OD ratio cut-off value of 0.4 .
Predicting neutralization activity is extremely important in selecting donors and recipients of convalescent plasma infusion therapy, which is a potential treatment for MERS-CoV infection [2]. In the present analysis, even after 21 dpoi, 19.5\% (8/41, Supplementary Table 1) of collected sera did not have neutralization activity, which emphasizes importance of antibody testing before collecting convalescent plasma. In addition, measurement 
a

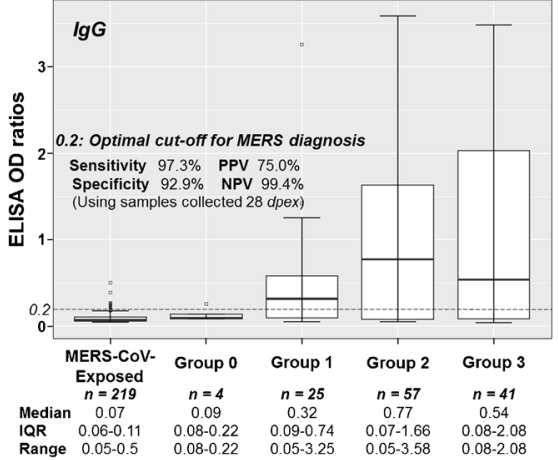

b

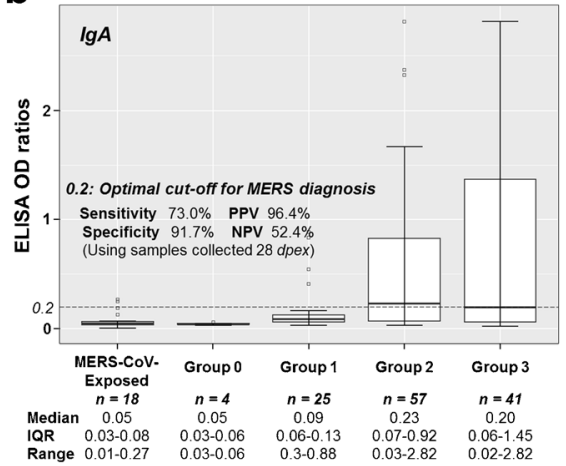

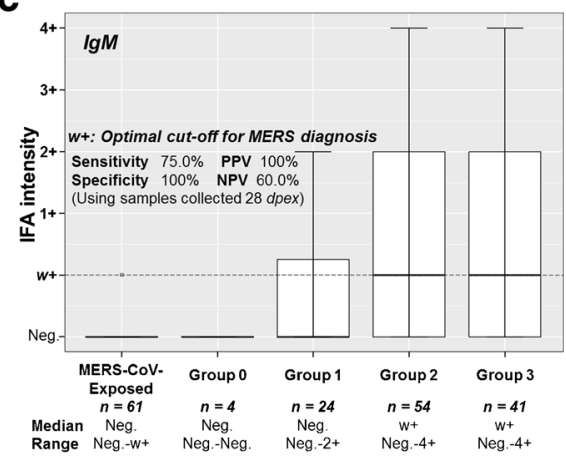

Fig. 2 Distribution of anti-MERS-CoV IgG, IgA, and IgM antibody values according to test population. (a) Distribution of anti-MERS-CoV ELISA IgG. (b) Distribution of anti-MERS-CoV ELISA IgA. (c) Distribution of anti-MERS-CoV IFA IgM. MERS-CoV-infected patients are classified according to the severity groups: asymptomatic infection (group 0), symptomatic infection without pneumonia (group 1), pneumonia without respiratory failure (group 2), and pneumonia progressing to respiratory failure (group 3 ). The distribution of antiMERS-CoV antibodies of group 0 patients was similar to those of rRTPCR-negative MERS-CoV-exposed individuals. Most values of MERS$\mathrm{CoV}$ non-infected patients were under the optimal cut-off value for MERS diagnosis. MERS-CoV Middle East respiratory syndrome coronavirus, ELISA enzyme-linked immunosorbent assay, $O D$ optical density, IFA immunofluorescence assay of neutralization activity is also important in selecting recipients and evaluating effect of convalescent plasma infusion. Using ELSIA tests with performance data from the present analysis, evaluation of neutralization activity can be performed in the field of MERS patient management without delay.

Diagnosing MERS-CoV infections using anti-MERS$\mathrm{CoV}$ serologic tests has been practically used for postexposure epidemiologic studies or sero-prevalence investigations in endemic regions $[3,6,12,16]$. To increase sensitivity of ELISA as a screening test, several epidemiologic studies applied low cut-off values of OD ratio, usually a three-fold value of negative controls (ELISA IgG OD ratios 0.2 to 0.3 , depending on study sites) [3, 6]. In the present analysis, we demonstrated that an ELISA IgG cut-off value of OD ratio 0.2 actually showed optimal performance with high specificity of 92 to $94 \%$. This is visually well demonstrated in Fig. 2; an ELISA IgG cut-off value of OD ratio 0.2 optimally discriminated negative controls and symptomatic infections (groups 1 to 3 ), while ELISA IgA and IFA IgM show inferior performance differentiating negative controls and mild patients (group 1). Asymptomatic patients (group 0) did not show any serologic responses, which make serologic diagnosis inapplicable. The manufacturer's instructions provided a significantly higher break point for MERS diagnosis to warrant specificity (for ELISA IgG, borderline OD ratio cut-off of $0.8-1.1$ and positive $\geq 1.1$ ) [7]. However, we demonstrated that ELISA IgG exhibits $100 \%$ specificity from the cut-off value of OD ratio 0.5 (Supplementary Table 5), while previous cut-off value of OD ratio 1.1 showed extremely low sensitivity of $34.8 \%$. Therefore, we suggest ELISA IgG OD ratio 0.2 as a new break point for MERS-CoV diagnosis for general application, while
OD ratio 0.5 can be applied for maximal specificity. In addition, we recommend taking at least a 28 -day interval from MERS-exposure to serum sampling for postexposure serologic investigations, to warrant seroconversion. In our unpublished data, seroconversion occurred around the third week of illness or the fourth week after exposure (18 dpoi in median, ranged 14-24; 22 dpex in median, ranged 18-30; Ko et al., data under review).

Although ELISA IgG showed slightly higher AUC than IFA IgG (Supplementary Table 4), the number of IFA tested samples was not sufficient, which limited comparison of performance between the two different methods. However, performance of ELISA IgG was at least not inferior to that of IFA IgG, which implies that additional confirmation by IFA after ELISA screening is not mandatory. In addition, considering the crossreactivity of the serologic tests to other coronaviruses [12], the performance for MERS diagnosis could be affected by the local epidemiology of human coronavirus infections.

In conclusion, in a performance analysis using 138 serum samples form 49 MERS-CoV-infected patients, ELISA IgG showed optimal performance in predicting neutralization activity and diagnosing MERS-CoV infection at cut-off values of OD ratio 0.4 and 0.2 , respectively. With this performance analysis, anti-MERS-CoV serologic tests can be practically used in the field of MERS management.

Acknowledgements We would like to express our sincerest condolences to the patients and families who suffered from the MERS outbreak. We also greatly appreciate the HCP and staff members at Samsung Medical Center and all other hospitals who worked together to overcome the MERS outbreak. Finally, we would like to thank Jinseob Kim for 
statistical advice and figure development, as well as Mingu Kang for IFA testing.

\section{Compliance with ethical standards}

Funding This work was supported by a Samsung Biomedical Research Institute (SBRI) grant [\#SMX1161321]. CD reports funding by EU grants Antigone (GA no. 278976) and Prepare (GA no. 602525).

Conflicts of interest There are no potential conflicts of interest relevant to this article to report.

Ethical approval This study was approved by the Institutional Review Board of Samsung Medical Center.

Informed consent As a retrospective study, the Institutional Review Board waived informed consent in the present study.

\section{References}

1. Zumla A, Hui DS, Perlman S (2015) Middle East respiratory syndrome. Lancet 386:995-1007. doi:10.1016/s0140-6736(15)60454-8

2. Arabi YM, Hajeer AH, Luke T, Raviprakash K, Balkhy H, Johani S, Al-Dawood A, Al-Qahtani S, Al-Omari A, Al-Hameed F, Hayden FG, Fowler R, Bouchama A, Shindo N, Al-Khairy K, Carson G, Taha Y, Sadat M, Alahmadi M (2016) Feasibility of using convalescent plasma immunotherapy for MERS-CoV infection, Saudi Arabia. Emerg Infect Dis 22:1554-1561. doi:10.3201/ eid2209.151164

3. Ko JH, Lee JY, Baek JY, Seok H, Park GE, Lee JY, Cho SY, Ha YE, Kang CI, Kang JM, Kim YJ, Kang ES, Kim SH, Jo IJ, Chung CR, Hahn MJ, Muller MA, Drosten C, Chung DR, Song JH, Peck KR (2017) Serologic evaluation of MERS screening strategy for healthcare personnel during a hospital-associated outbreak. Infect Control Hosp Epidemiol 38:234-238. doi:10.1017/ice.2016.251

4. Park WB, Perera RA, Choe PG, Lau EH, Choi SJ, Chun JY, Oh HS, Song KH, Bang JH, Kim ES, Kim HB, Park SW, Kim NJ, Man Poon LL, Peiris M, Oh MD (2015) Kinetics of serologic responses to MERS coronavirus infection in humans, South Korea. Emerg Infect Dis 21:2186-2189. doi:10.3201/eid2112.151421

5. Corman VM, Albarrak AM, Omrani AS, Albarrak MM, Farah ME, Almasri M, Muth D, Sieberg A, Meyer B, Assiri AM, Binger T, Steinhagen K, Lattwein E, Al-Tawfiq J, Muller MA, Drosten C, Memish ZA (2016) Viral shedding and antibody response in 37 patients with Middle East respiratory syndrome coronavirus infection. Clin Infect Dis 62:477-483. doi:10.1093/cid/civ951

6. Muller MA, Meyer B, Corman VM, Al-Masri M, Turkestani A, Ritz D, Sieberg A, Aldabbagh S, Bosch BJ, Lattwein E, Alhakeem RF, Assiri AM, Albarrak AM, Al-Shangiti AM, AlTawfiq JA, Wikramaratna P, Alrabeeah AA, Drosten C, Memish ZA (2015) Presence of Middle East respiratory syndrome coronavirus antibodies in Saudi Arabia: a nationwide, cross-sectional, serological study. Lancet Infect Dis 15:559-564. doi:10.1016/s14733099(15)70090-3
7. Euroimmun (2014) Anti-MERS-CoV ELISA (IgG) Test instruction, EUROIMMUN, Lübeck, Germany, El 2604G A UK C01.doc, Version: 06/10/2014

8. Park GE, Ko JH, Peck KR, Lee JY, Lee JY, Cho SY, Ha YE, Kang CI, Kang JM, Kim YJ, Huh HJ, Ki CS, Lee NY, Lee JH, Jo IJ, Jeong BH, Suh GY, Park J, Chung CR, Song JH, Chung DR (2016) Control of an outbreak of Middle East respiratory syndrome in a tertiary Hospital in Korea. Ann Intern Med 165:87-93. doi:10. 7326/m15-2495

9. Ko JH, Park GE, Lee JY, Lee JY, Cho SY, Ha YE, Kang CI, Kang JM, Kim YJ, Huh HJ, Ki CS, Jeong BH, Park J, Chung CR, Chung DR, Song JH, Peck KR (2016) Predictive factors for pneumonia development and progression to respiratory failure in MERS-CoV infected patients. J Inf Secur 73:468-475. doi:10.1016/j.jinf.2016.08.005

10. Madani TA (2014) Case definition and management of patients with MERS coronavirus in Saudi Arabia. Lancet Infect Dis 14: 911-913. doi:10.1016/s1473-3099(14)70918-1

11. Corman VM, Eckerle I, Bleicker T, Zaki A, Landt O, EschbachBludau M, van Boheemen S, Gopal R, Ballhause M, Bestebroer TM, Muth D, Muller MA, Drexler JF, Zambon M, Osterhaus AD, Fouchier RM, Drosten C (2012) Detection of a novel human coronavirus by real-time reverse-transcription polymerase chain reaction. Euro Surveill 17

12. Drosten C, Meyer B, Muller MA, Corman VM, Al-Masri M, Hossain R, Madani H, Sieberg A, Bosch BJ, Lattwein E, Alhakeem RF, Assiri AM, Hajomar W, Albarrak AM, Al-Tawfiq JA, Zumla AI, Memish ZA (2014) Transmission of MERScoronavirus in household contacts. N Engl J Med 371:828-835. doi:10.1056/NEJMoa1405858

13. Raj VS, Mou H, Smits SL, Dekkers DH, Muller MA, Dijkman R, Muth D, Demmers JA, Zaki A, Fouchier RA, Thiel V, Drosten C, Rottier PJ, Osterhaus AD, Bosch BJ, Haagmans BL (2013) Dipeptidyl peptidase 4 is a functional receptor for the emerging human coronavirus-EMC. Nature 495:251-254. doi:10.1038/ nature 12005

14. Muth D, Corman VM, Meyer B, Assiri A, Al-Masri M, Farah M, Steinhagen K, Lattwein E, Al-Tawfiq JA, Albarrak A, Muller MA, Drosten C, Memish ZA (2015) Infectious Middle East respiratory syndrome coronavirus excretion and serotype variability based on live virus isolates from patients in Saudi Arabia. J Clin Microbiol 53:2951-2955. doi:10.1128/jcm.01368-15

15. Corman VM, Muller MA, Costabel U, Timm J, Binger T, Meyer B, Kreher P, Lattwein E, Eschbach-Bludau M, Nitsche A, Bleicker T, Landt O, Schweiger B, Drexler JF, Osterhaus AD, Haagmans BL, Dittmer U, Bonin F, Wolff T, Drosten C (2012) Assays for laboratory confirmation of novel human coronavirus (hCoV-EMC) infections. Euro Surveill 17

16. Kim CJ, Choi WS, Jung Y, Kiem S, Seol HY, Woo HJ, Choi YH, Son JS, Kim KH, Kim YS, Kim ES, Park SH, Yoon JH, Choi SM, Lee H, Oh WS, Choi SY, Kim NJ, Choi JP, Park SY, Kim J, Jeong SJ, Lee KS, Jang HC, Rhee JY, Kim BN, Bang JH, Lee JH, Park S, Kim HY, Choi JK, Wi YM, Choi HJ (2016) Surveillance of the Middle East respiratory syndrome (MERS) coronavirus $(\mathrm{CoV})$ infection in healthcare workers after contact with confirmed MERS patients: incidence and risk factors of MERS-CoV seropositivity. Clin Microbiol Infect 22:880-886. doi:10.1016/j.cmi.2016.07.017 